\title{
Pilot Beam Sequence Design for Channel Estimation in Millimeter-Wave MIMO Systems: A POMDP Framework
}

\author{
Junyeong Seo, Student Member, IEEE, Youngchul Sung, Senior Member, IEEE \\ Gilwon Lee, and Donggun Kim, Student Members, IEEE
}

\begin{abstract}
In this paper, adaptive pilot beam sequence design for channel estimation in large millimeter-wave (mmWave) MIMO systems is considered. By exploiting the sparsity of mmWave MIMO channels with the virtual channel representation and imposing a Markovian random walk assumption on the physical movement of the line-of-sight (LOS) and reflection clusters, it is shown that the sparse channel estimation problem in large mmWave MIMO systems reduces to a sequential detection problem that finds the locations and values of the non-zerovalued bins in a two-dimensional rectangular grid, and the optimal adaptive pilot design problem can be cast into the framework of a partially observable Markov decision process (POMDP). Under the POMDP framework, an optimal adaptive pilot beam sequence design method is obtained to maximize the accumulated transmission data rate for a given period of time. Numerical results are provided to validate our pilot signal design method and they show that the proposed method yields good performance.
\end{abstract}

Index Terms-Millimeter-wave, Large MIMO, Channel estimation, Partially Observable Markov Processes (POMDP)

\section{INTRODUCTION}

Millimeter-wave (mmWave) communication is rising as a key technology to provide high data rates with wide bandwidth (BW) in 5G wireless systems. However, the signal pathloss in the mmWave band is much larger than that in the lower band currently used in most wireless access networks. To overcome the pathloss, there is on-going research about highly directional beamforming techniques in mmWave systems using large antenna arrays [1]-[3]. Typically these beamforming techniques require channel state information (CSI) at the transmitter and the receiver, but it is more difficult to obtain CSI in the mmWave band than in the lower band because of the high propagation directivity and the low signal-to-noise ratio (SNR) before beamforming. Thus, the accurate and efficient channel estimation is important to attain the promised BW gain of the mmWave band.

One of the major differences between the channels in conventional MIMO systems in lower bands and large mmWave MIMO systems is the sparsity in the MIMO channel. Whereas channel estimation methods in conventional lowerband MIMO systems assume rich scattering or the knowledge of the channel covariance matrix in the rank-deficient case, such assumptions are not valid in the mmWave band [4].

The authors are with the Dept. of Electrical Engineering, KAIST, Daejeon 305-701, South Korea. E-mail:\{jyseo@, ysung@ee., gwlee@, and dg.kim@\}kaist.ac.kr. This work was supported by ICT R\&D program of MSIP/IITP [ 11-911-04-001, Development of Adaptive Beam Multiple Access Technology without Interference based on Antenna Node Grouping].
Among many possible ray directions resolved by a large antenna array, only a few directions actually carry the signal, and these signal-carrying directions are unknown beforehand [4]. To tackle the challenge of the sparse channel estimation in the mmWave band, algorithms based on compressed sensing (CS) have recently been developed [2]-[5]. In [4], the channel estimation problem is formulated by capturing the sparse nature of the channel, and CS techniques are used to analyze the sparse channel estimation performance. Recently, an efficient channel estimation and training beam design method for large mmWave MIMO systems was proposed based on adaptive CS in [2], [3]. In the proposed method, the channel estimation is conducted over multiple slots under the assumption that the channel does not vary over the considered multiple slots. Each slot consists of multiple training beam symbol times so that the sparse recovery is feasible at each slot, and the training beam at the next slot is adaptively designed depending on the previous slot observation based on a space bisection approach [2], [3]. Such a design strategy is a reasonable choice to search the actual signal-carrying directions in the space. The channel estimation problem in time-varying sparse MIMO systems was also considered in [4], [6], where only the time-variation of the complex path gains was considered in [4] and the time variation of AoDs and AoAs as well as the complex path gains were considered in [6].

In this paper, we consider the adaptive pilot beam sequence design to estimate the sparse channel in large mmWave MIMO systems based on a decision-theoretical approach, and propose a strategy to design the pilot beam sequence that is optimal in a certain sense. Exploiting the sparsity of mmWave channels with the virtual channel representation and imposing a Markovian random walk assumption on the physical movement of the LOS and reflection clusters, we cast the sparse channel estimation problem in large mmWave MIMO systems into the framework of a partially observable Markov decision process (POMDP) with finite horizon [7], where we need to find the locations and values of the non-zero-valued bins in a twodimensional rectangular grid. Under the proposed POMDP framework, we derive an optimal adaptive pilot beam sequence design method to maximize the accumulated transmission data rate for a given period of time.

\section{System MOdeL}

\section{A. Sparse Channel Modeling in mmWave Systems}

We consider a mmWave MIMO system with a uniform linear array (ULA) of $N_{t}$ antennas at the transmitter and an 
ULA of $N_{r}$ antennas at the receiver. The received signal at symbol time $n$ is given by

$$
\mathbf{y}_{n}=\mathbf{H}_{n} \mathbf{x}_{n}+\mathbf{n}_{n}, \quad n=1,2, \cdots,
$$

where $\mathbf{H}_{n}$ is the $N_{r} \times N_{t}$ MIMO channel matrix at time $n$, $\mathbf{x}_{n}$ is the $N_{t} \times 1$ transmitted symbol vector at time $n$ with a power constraint $\mathbb{E}\left\{\mathbf{x}_{n} \mathbf{x}_{n}^{H}\right\} \leq P_{t}$, and $\mathbf{n}_{n}$ is the $N_{r} \times 1$ Gaussian noise vector at time $n$ from $\mathcal{C N}\left(\mathbf{0}, \sigma_{w}^{2} \mathbf{I}_{N_{r}}\right)$. The MIMO channel matrix $\mathbf{H}_{n}$ can be expressed in terms of the physical propagation paths as

$$
\mathbf{H}_{n}=\sqrt{N_{t} N_{r}} \sum_{\ell=1}^{L} \alpha_{n, \ell} \mathbf{a}_{R X}\left(\theta_{n, \ell}^{r}\right) \mathbf{a}_{T X}^{H}\left(\theta_{n, \ell}^{t}\right),
$$

where $\alpha_{n, \ell} \sim \mathcal{C N}\left(0, \xi^{2}\right)$ is the complex gain of the $\ell$-th path at time $n$, and $\theta_{n, \ell}^{r}$ and $\theta_{n, \ell}^{t}$ are the angle-of-arrival (AoA) and angle-of-departure (AoD) normalized directions of the $\ell$-th path at time $n$ for the receiver and the transmitter, respectively. Here, the normalized direction $\theta$ is related to the physical angle $\phi \in[-\pi / 2, \pi / 2]$ as $\theta=\frac{d \sin (\phi)}{\lambda}$, where $d$ is the spacing between two adjacent antennas and $\lambda$ is the signal wavelength (we assume $\frac{d}{\lambda}=\frac{1}{2}$ ), and $\mathbf{a}_{R X}\left(\theta^{r}\right)$ and $\mathbf{a}_{T X}\left(\theta^{t}\right)$ are the receiver response and the transmitter steering vector, which are defined as [8]

$$
\begin{aligned}
& \mathbf{a}_{R X}\left(\theta^{r}\right)=\frac{1}{\sqrt{N_{r}}}\left[1, e^{-\iota 2 \pi \theta^{r}}, \cdots, e^{-\iota\left(N_{r}-1\right) 2 \pi \theta^{r}}\right]^{T}, \\
& \mathbf{a}_{T X}\left(\theta^{t}\right)=\frac{1}{\sqrt{N_{t}}}\left[1, e^{-\iota 2 \pi \theta^{t}}, \cdots, e^{-\iota\left(N_{t}-1\right) 2 \pi \theta^{t}}\right]^{T} .
\end{aligned}
$$

With neglecting the angle quantization error the physical MIMO channel matrix $\mathbf{H}_{n}$ can be rewritten in terms of the virtual channel matrix $\mathbf{H}_{n}^{V}$ [9]:

$$
\mathbf{H}_{n}=\mathbf{A}_{R} \mathbf{H}_{n}^{V} \mathbf{A}_{T}^{H}
$$

where $\mathbf{A}_{R}=\left[\mathbf{a}_{R X}\left(\tilde{\theta}_{1}^{r}\right), \cdots, \mathbf{a}_{R X}\left(\tilde{\theta}_{N_{r}}^{r}\right)\right], \tilde{\theta}_{i}^{r}=-\frac{1}{2}+\frac{i-1}{N_{r}}$ for $i=1, \cdots, N_{r}$, and $\mathbf{A}_{T}=\left[\mathbf{a}_{T X}\left(\tilde{\theta}_{1}^{t}\right), \cdots, \mathbf{a}_{T X}\left(\tilde{\theta}_{N_{t}}^{t}\right)\right], \tilde{\theta}_{j}^{t}=$ $-\frac{1}{2}+\frac{j-1}{N_{t}}$ for $j=1, \cdots, N_{t}$. The element in the $i$-th row and the $j$-th column of $\mathbf{H}_{n}^{V}$ indicates the complex channel gain whose AoA and AoD normalized directions are $\tilde{\theta}_{i}^{r}$ and $\tilde{\theta}_{j}^{t}$, respectively. The sparsity in the physical channel model in (2) is translated into the constraint that $\mathbf{H}_{n}^{V}$ has only $L$ non-zero elements (i.e. $\left.\sum_{j=1}^{N_{t}}\left\|\mathbf{H}_{n}^{V}(:, j)\right\|_{0}=L\right)$ and $L \ll N_{t} N_{r}$ for large $N_{t}$.

We assume that the receiver has $N_{r}\left(\ll N_{t}\right)$ RF chains so that it can implement the filter bank $\mathbf{A}_{R}^{H}$ to look ahead for all possible AoA directions. In this case, the receiver filter-bank output is given by

$$
\mathbf{y}_{n}^{\prime}:=\mathbf{A}_{R}^{H} \mathbf{y}_{n}=\mathbf{H}_{n}^{V} \mathbf{A}_{T}^{H} \mathbf{x}_{n}+\mathbf{n}_{n}^{\prime}
$$

where $\mathbf{n}_{n}^{\prime}=\mathbf{A}_{R}^{H} \mathbf{n}_{n}$.

By simply transmitting a pilot beam sequence $\mathbf{a}_{T X}\left(\tilde{\theta}_{1}^{t}\right)$, $\mathbf{a}_{T X}\left(\tilde{\theta}_{2}^{t}\right), \cdots, \mathbf{a}_{T X}\left(\tilde{\theta}_{N_{t}}^{t}\right)$, the receiver can estimate the positions and values of the $L$ non-zero elements of $\mathbf{H}_{n}^{V}$ if the channel is time-invariant for $N_{t}$ symbol times. However, such a method does not exploit the channel sparsity and/or the channel dynamic, and is inefficient when $N_{t}$ is large.

\section{B. The Proposed Dynamic Channel Model}

To design a very efficient pilot beam sequence, we exploit the channel dynamic, and model the channel dynamic by using a Markovian structure that is different from the Gauss-Markov or state-space channel model conventionally used to model the channel dynamic. In large mmWave MIMO systems, the sparsity should be captured in the channel dynamic. Here, we focus on the locations of the non-zero elements of $\mathbf{H}_{n}^{V}$ rather than the values, since the value will be obtained with reasonable quality once the correct direction is hit by the pilot beam with high power. Note that each propagation path is generated by either LOS or a reflection cluster and the physical movement of the receiver or a reflection cluster can be modelled as a random walk in space. This random walk translates into each nonzero bin's random walk in the virtual channel matrix. Thus, we assume a stationary block Markovian random walk for the dynamic of the virtual channel matrix. That is, the virtual channel matrix $\mathbf{H}_{(k)}^{V}$ at slot $k$ is constant over the slot and changes to $\mathbf{H}_{(k+1)}^{V}$ at the next slot $k+1$ with the aforementioned random walk with a set of transition probabilities. We also assume that the movement of each path is independent. Since the receiver checks all possible AoA directions in parallel, we here only consider the random walk across AoD, i.e., the column-wise movement of each non-zero bin in the virtual channel matrix.

1) The Single Path Case: First, consider the single path case, i.e., $L=1$. The single path (or non-zero bin) is located in a certain column of the virtual channel matrix at slot $k$, and stay at the same column or moves to another column of the virtual channel matrix at slot $k+1$ according to the explained random walk. Since we have $N_{t}$ columns in the virtual channel matrix, the number $N$ of states for $L=1$ is $N_{t}$. Let us denote the set of all possible states by $\mathcal{S}=\left\{1,2, \cdots, N_{t}\right\}$, where state $i$ denotes the state that the path is located in the $i$-th column of the virtual channel matrix. With the set $\mathcal{S}$ of states defined, the $(i, j)$-th element of the $N \times N$ state transition probability matrix $\mathbf{P}$ is given by

$$
p_{i j}=\operatorname{Pr}\left\{S_{k+1}=j \mid S_{k}=i\right\}, \quad i, j \in \mathcal{S},
$$

where $S_{k}$ and $S_{k+1}$ denote the states of slots $k$ and $k+1$, respectively. The transition probability matrix captures the characteristics of the path's movement behavior and thus it should carefully be designed by considering the physics of the receiver and reflection cluster movement, e.g., vehicular channels or pedestrian channels. In the extreme case of a static channel, we have $\mathbf{P}=\mathbf{I}$, i.e., an identity matrix.

2) The Multiple Path Case: Now consider the multiple propagation path case, i.e., $L \geq 2$. In this case, each path (or non-zero bin) is located in a column of the virtual channel matrix. The set $\mathcal{S}$ of all possible states is now given by $\mathcal{S}=$ $\left\{\left(i_{1}, i_{2}, \cdots, i_{L}\right), i_{1}, i_{2}, \cdots, i_{L}=1,2, \cdots, N_{t}\right\}$, where state $\left(i_{1}, \cdots, i_{\ell}, \cdots, i_{L}\right)$ denotes that the $\ell$-th path is located at the $i_{\ell}$-th column of the virtual channel matrix for $\ell=1, \cdots, L$ and the cardinality $N$ of $\mathcal{S}$ is $N_{t}^{L}$. For notational simplicity, let us also use the following notation: 

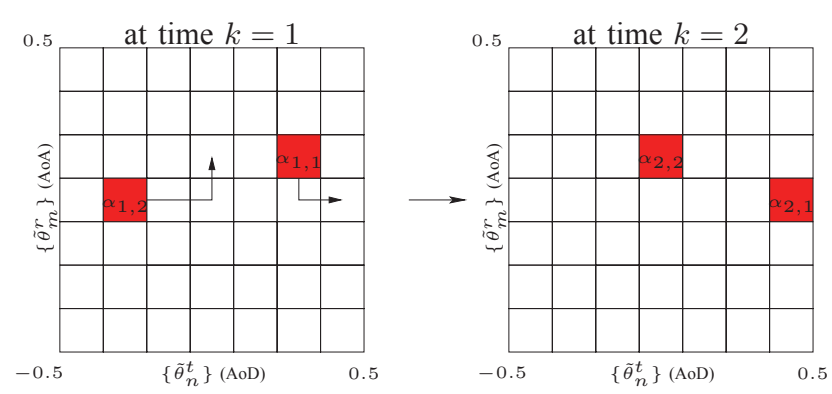

Fig. 1. An illustration of a transition of each path when $L=2$ and $N_{t}=$ $N_{r}=7$.

$$
\mathcal{S}=\left\{\mathbf{s}^{(1)}, \mathbf{s}^{(2)}, \cdots, \mathbf{s}^{(N)}\right\},
$$

where states $\left(i_{1}, \cdots, i_{L}\right), i_{1}, \cdots, i_{L}=1, \cdots, N_{t}$, are enumerated into states $\mathbf{s}^{(i)}, i=1,2, \cdots, N=N_{t}^{L}$. We here allow multiple paths can merge on and diverge from a column of the virtual matrix. Then the state transition probability in the $L$ independent path case is given by

$$
\begin{aligned}
& \operatorname{Pr}\left\{S_{k+1}=\left(j_{1}, \cdots, j_{L}\right) \mid S_{k}=\left(i_{1}, \cdots, i_{L}\right)\right\} \\
& \quad=p_{i_{1} j_{1}} p_{i_{2} j_{2}} \times \cdots \times p_{i_{L} j_{L}},
\end{aligned}
$$

where $p_{i j}$ denotes the transition probability that a path moves from the $i$-th column to the $j$-th column of the virtual channel matrix at the next slot and is defined in (7).

Fig. 1 illustrates an example of transitions of the paths when $L=2$ and $N_{t}=N_{r}=7$. The transition probability of Fig. 1 is $p_{67} \times p_{24}$ due to the independence assumption for each path.

\section{Channel Sensing with Pilot Beam Sequence}

We assume that $M_{p}\left(L \leq M_{p} \ll N_{t}\right)$ symbol times in each slot are used for transmitting a sequence of pilot beams and one column of $\mathbf{A}_{T}$ is selected as the pilot beam in each pilot symbol time. (We assume that highly directional pilot beam is required to obtain a channel gain estimate with reasonable quality due to large pathloss in the mmWave band.) Hence, $M_{p}$ columns of $\mathbf{A}_{T}$ are selected as the pilot beam sequence for the $M_{p}$ pilot symbol times per slot. If $\mathbf{a}_{T X}\left(\tilde{\theta}_{i_{m}}^{t}\right)$ is transmitted as the pilot signal at the $m$-th pilot symbol time in the $k$-th slot, from (6), the receiver filter-bank output is given by

$$
\mathbf{y}_{(k)}^{\prime}[m]=\mathbf{H}_{(k)}^{V}\left(:, i_{m}\right)+\mathbf{n}_{(k)}^{\prime}[m],
$$

where $\mathbf{y}_{(k)}^{\prime}[m]$ denotes the receiver filter-bank output at symbol time $m$ of slot $k, \mathbf{n}_{(k)}^{\prime}[m]$ is similarly defined, and $\mathbf{H}_{(k)}^{V}\left(:, i_{m}\right)$ denotes the $i_{m}$-th column of $\mathbf{H}_{(k)}^{V}$. After the transmission of the sequence of pilot beams for one slot is finished, the receiver senses and estimates the $M_{p}$ columns of $\mathbf{H}_{(k)}^{V}$ corresponding to the $M_{p}$ pilot beams. Then, the receiver feeds back the sensing results and estimated channel gains corresponding to the $M_{p}$ pilot beam directions to the transmitter. The process is depicted in Fig. 2.

Now, the problem is to design the sequence of pilot beams for $M_{p}$ symbol times for each slot in a certain optimal way.

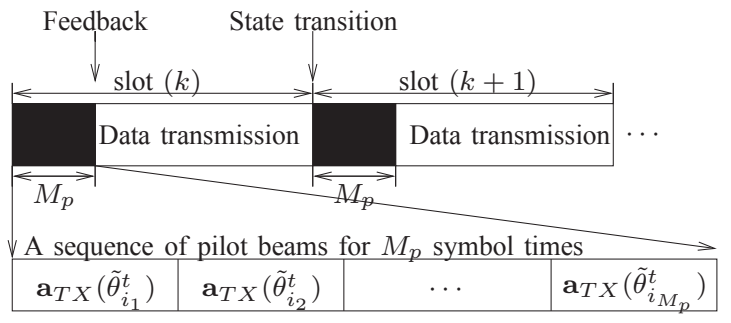

Fig. 2. The process of the pilot training.

Since $M_{p} \ll N_{t}$, we can only sense a few columns of $\mathbf{H}_{(k)}^{V}$ at slot $k$. Therefore, $M_{p}$ pilot beams at each slot should be designed judiciously by exploiting the channel dynamic and the available information in all the previous slots.

\section{POMdP Formulation for Pilot BeAm Design FOR SPARSE CHANNEL ESTIMATION}

\section{A. Action Space at the Transmitter and Feedback from the} Receiver

In Section II, we assumed that $M_{p}$ columns of $\mathbf{A}_{T}$ are selected as the pilot beam sequence for the $M_{p}$ pilot symbols per slot. This is equivalent to choosing $M_{p}$ columns of the virtual channel matrix at each slot to be sensed by the pilot beam sequence. We denote the selected column indices of the virtual channel matrix by $\mathbf{a}=\left[a_{1}, a_{2}, \cdots, a_{M_{p}}\right]$, where $a_{m}$ indicates the index of the column of the virtual channel matrix that is sensed at symbol time $m$. (a is referred to as the action vector.) Hence, there are $\left(\begin{array}{l}N_{t} \\ M_{p}\end{array}\right)$ possible a's and the optimal pilot beam sequence design problem reduces to choosing the best $\mathbf{a}$ at each slot.

After the chosen pilot beam sequence is transmitted to the receiver, the receiver feeds back the result of detection to the transmitter for pilot beam sequence design for the next slot. The feedback information contains the information about the existence* of paths in the selected columns of the virtual channel matrix as well as the complex gains of the detected paths. Then, the transmitter uses the channel gain information of the detected paths for beamforming during the data transmission period and uses the feedback information about the existence of paths to choose the pilot beam sequence for the next slot in an adaptive manner. The latter feedback information can be modeled as $\mathbf{o}=\left[o_{1}, o_{2}, \cdots, o_{M_{p}}\right] \in\{0,1\}^{M_{p}}$, where $o_{m}=1$ indicates that a path is detected by the pilot beam transmitted at the $m$-th pilot symbol time, and otherwise $o_{m}=0$. Since there exist $2^{M_{p}}$ possibilities in $\mathbf{o}$, the feedback information space is defined as $\mathcal{O}=\left[\mathbf{o}^{(1)}, \mathbf{o}^{(2)}, \cdots, \mathbf{o}^{\left(2^{M_{p}}\right)}\right]$. When the current state of the virtual channel matrix is $\mathbf{s}^{(i)}$ and the action vector $\mathbf{a}$ is selected for the pilot beam sequence for the current slot, the probability that the transmitter observes the feedback information $\mathbf{o}^{(j)}$ is denoted as $q_{i j}^{\mathbf{a}}$, i.e.,

$$
q_{i j}^{\mathbf{a}} \triangleq \operatorname{Pr}\left\{\mathbf{o}=\mathbf{o}^{(j)} \mid \mathbf{s}^{(i)}, \mathbf{a}\right\} \quad \text { for } \mathbf{s}^{(i)} \in \mathcal{S}, \mathbf{o}^{(j)} \in \mathcal{O} .
$$

* The detection can be wrong. This is another reason for POMDP in addition to the limited search of $M_{p}$ columns out of the $N_{t}$ total columns per slot. 
This probability depends on the detector used to identify the existence of a non-zero bin in a column at the receiver.

\section{B. Sufficient Statistic}

At the beginning of slot $k$, the information from all the past slot pilot beam sequences and feedback information can be summarized as a belief vector: ${ }^{\dagger}$

$$
\boldsymbol{\pi}_{k}=\left[\pi_{k, 1}, \pi_{k, 2}, \cdots, \pi_{k, N}\right],
$$

where $\pi_{k, i}$ is the probability that the state at the beginning of slot $k$ is state $\mathbf{s}^{(i)}$ conditioned on all past pilot beam sequences and feedback information. It is known that the belief vector is a sufficient statistic for the action, i.e., the design of the optimal pilot beam sequence for slot $k$ [10]. The transmitter uses the belief vector to optimally choose the pilot beam sequence for slot $k$ that maximizes the expected reward, and updates the belief vector for the next block based on new feedback information.

\section{The Reward and The Policy}

A reward is gained during the data transmission period according to the accuracy of channel estimation. According to the objective, there can be several ways to define the reward. Since we want to track all actual propagation paths in the sparse mmWave MIMO channel successfully, we define the reward for each slot as the number of actual propagation paths (i.e., the number of non-zero bins in the virtual channel matrix) detected by the selected pilot beam sequence. Since the state at slot $k$ and the feedback information are unknown at the time of action, we should consider the expected reward [7]. If the state of the virtual channel matrix prior to the state transition at slot $k$ is $\mathbf{s}^{(n)}$, then the immediate expected reward at slot $k$ can be expressed as

$$
R\left(\mathbf{s}^{(n)}, \mathbf{a}\right)=\sum_{i=1}^{N} p_{n i} \sum_{j=1}^{2^{M_{p}}} q_{i j}^{\mathbf{a}} \sum_{m=1}^{M_{p}} N_{\mathbf{s}^{(i)}, a_{m}}^{B I N} o_{m}^{(j)},
$$

where $N_{\mathbf{S}^{(i)}, a_{m}}^{B I N}$ is the number of non-zero bins in column $a_{m}$ when the virtual channel matrix is in state $\mathbf{s}^{(i)}, o_{m}^{(j)}$ is the $m$-th element of $\mathbf{o}^{(j) \ddagger}$, and the state transition from $\mathbf{s}^{(n)}$ to all possible $\mathbf{s}^{(i)}$ within the slot is captured by $\sum_{i=1}^{N} p_{n i}(\cdot)$. Here, the false alarm of the receiver detector does not affect the immediate reward because in this case $o_{m}^{(j)}=1$ but $N_{\mathbf{s}^{(i)}, a_{m}}^{B I N}=$ 0 . In the case of miss detection, the opportunity is simply lost.

When the belief vector $\pi_{k}$ is given at the beginning of slot $k$ and $S_{k}$ is the random variable representing the state at the beginning of the slot, the immediate expected reward at slot $k$ is given by

$$
\begin{aligned}
\mathcal{R}\left(\boldsymbol{\pi}_{k}, \mathbf{a}\right) & =\mathbb{E}\left\{R\left(S_{k}, \mathbf{a}\right) \mid \boldsymbol{\pi}_{k}\right\} \\
& =\left\langle\mathbf{R}(\mathbf{a}), \boldsymbol{\pi}_{k}\right\rangle
\end{aligned}
$$

where $\mathbf{R}(\mathbf{a})=\left[R\left(\mathbf{s}^{(1)}, \mathbf{a}\right), R\left(\mathbf{s}^{(2)}, \mathbf{a}\right), \cdots, R\left(\mathbf{s}^{(N)}, \mathbf{a}\right)\right]$, and $\langle\cdot, \cdot\rangle$ denotes the inner product operation.

In the POMDP framework, a policy $\delta$ is defined as a sequence of functions that maps the belief vector to an action

\footnotetext{
${ }^{\dagger}$ Note that the belief vector $\pi_{k}$ is conventionally defined prior to the state transition for each slot. The belief vector after the state transition can simply be updated by using the state transition probability matrix.

$\ddagger$ Two paths with the same AoD and different AoAs are considered as two different paths.
}

for each slot [10], [11], where the action in our formulation is the choice of the pilot beam sequence. The optimal policy is the policy that maximizes the total immediate expected reward over $T$ slots $^{\S}$ when the initial belief vector $\boldsymbol{\pi}_{1}$ at the beginning of the transmission is given. In other words, the optimal policy $\delta^{*}$ is expressed as

$$
\delta^{*}=\underset{\delta}{\arg \max } \mathbb{E}_{\delta}\left[\sum_{k=1}^{T} R\left(S_{k}, \mathbf{a}_{k}\right) \mid \boldsymbol{\pi}_{1}\right],
$$

where $\mathbf{a}_{k}$ is the action vector at slot $k, \mathbb{E}_{\delta}$ is the conditional expectation when the policy $\delta$ is given, and $T$ is the total number of slots.

\section{The Optimal and Suboptimal Strategies for CHANNEL ESTIMATION}

Under the proposed formulation the optimal pilot beam sequence design problem is equivalent to the problem of finding the optimal policy that satisfies (15). When the initial belief vector $\pi_{1}$ is given at $k=1$, we can define an optimal value function $V\left(\boldsymbol{\pi}_{1}\right)$ as the maximum total expected reward by the optimal policy $\delta^{*}$ :

$$
V\left(\boldsymbol{\pi}_{1}\right)=\mathbb{E}_{\delta^{*}}\left[\sum_{k=1}^{T} R\left(S_{k}, \mathbf{a}_{k}\right) \mid \boldsymbol{\pi}_{1}\right] .
$$

Now consider $V^{k}\left(\boldsymbol{\pi}_{k}\right)$ defined as the maximum remaining expected reward that can be obtained from slot $k$ to slot $T$ when a belief vector $\pi_{k}$ is given at the beginning of slot $k$. Then, $V^{k}\left(\boldsymbol{\pi}_{k}\right)$ can be decomposed as [12]

$$
\begin{aligned}
& V^{k}\left(\boldsymbol{\pi}_{k}\right) \\
& =\max _{\mathbf{a}_{k}}\left\{\left\langle\mathbf{R}\left(\mathbf{a}_{k}\right), \boldsymbol{\pi}_{k}\right\rangle+\sum_{j=1}^{2^{M_{p}}} V^{k+1}\left(\mathcal{T}\left(\boldsymbol{\pi}_{k} \mid \mathbf{o}^{(j)}, \mathbf{a}_{k}\right)\right) \gamma\left(\mathbf{o}^{(j)} \mid \boldsymbol{\pi}_{k}, \mathbf{a}_{k}\right)\right\},
\end{aligned}
$$

where $\gamma\left(\mathbf{o}^{(j)} \mid \boldsymbol{\pi}_{k}, \mathbf{a}_{k}\right)=\sum_{i=1}^{N} q_{i j}^{\mathbf{a}_{k}} \sum_{n=1}^{N} \pi_{k, n} p_{n i}$ and $\mathcal{T}\left(\boldsymbol{\pi}_{k} \mid \mathbf{o}^{(j)}, \mathbf{a}_{k}\right)$ is the updated belief vector from $\boldsymbol{\pi}_{k}$ at slot $k$ for the next slot after taking action $\mathbf{a}_{k}$ and observing $\mathbf{o}^{(j)}$. $\mathcal{T}\left(\boldsymbol{\pi}_{k} \mid \mathbf{o}^{(j)}, \mathbf{a}_{k}\right)$ can easily be computed using Bayes's formula as [10], [11]

$$
\boldsymbol{\pi}_{k+1}=\left[\pi_{k+1,1}, \pi_{k+1,2}, \cdots, \pi_{k+1, N}\right]=\mathcal{T}\left(\boldsymbol{\pi}_{k} \mid \mathbf{o}^{(j)}, \mathbf{a}_{k}\right),
$$

where the $i$-th element of $\boldsymbol{\pi}_{k+1}$ is given by

$$
\begin{aligned}
\pi_{k+1, i} & =\operatorname{Pr}\left\{\mathbf{s}_{k}=\mathbf{s}^{(i)} \mid \mathbf{o}^{(j)}, \mathbf{a}_{k}, \boldsymbol{\pi}_{k}\right\} \\
& =\frac{q_{i j}^{\mathbf{a}_{k}} \sum_{n=1}^{N} \pi_{k, n} p_{n i}}{\sum_{i=1}^{N} q_{i j}^{\mathbf{a}_{k}} \sum_{n=1}^{N} \pi_{k, n} p_{n i}} .
\end{aligned}
$$

The optimal policy $\delta^{*}$ over the considered transmission period $k=[1,2, \cdots, T]$ can be computed via the recursion (17) from backward once the state transition probability $\mathbf{P}$, reward, and observation and action spaces are given [10], [11], [13]. This computation can be done off-line and the optimal policy can be stored beforehand. Then, in actual transmission,

\footnotetext{
§uch a formulation is called a finite-horizon POMDP. The formulation here can be modified to the infinite-horizon case.

I Hence, for each channel type, we can pre-compute the policy and store it. This is one of the main advantages of the proposed pilot beam design approach.
} 


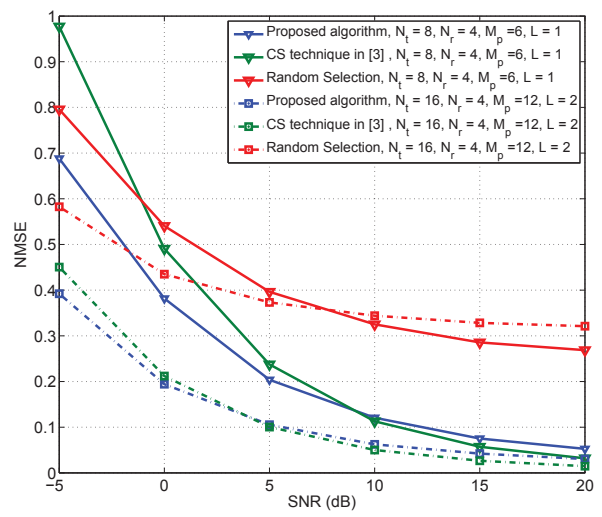

Fig. 3. NMSE performance comparison for $\left(N_{t}=8, N_{r}=4, M_{p}=\right.$ $6, L=1)$ and $\left(N_{t}=16, N_{r}=4, M_{p}=12, L=2\right)$

we start from $k=1$ with $\pi_{1}$ and repeat action and observation. The complexity of this actual operation is insignificant.

As the number of states and the size of the action space increase, obtaining the optimal policy for the POMDP problem requires high complexity. To reduce the computational complexity, we can use point-based POMDP value iteration algorithms proposed in [14]-[16]. Alternatively, to reduce the complexity, we can simply use the greedy policy that considers only the immediate expected reward at each slot.

\section{NumERicAl RESUlts}

In this section, we provide some numerical results to evaluate the performance of the proposed pilot beam design and channel estimation method for sparse mmWave channels. Throughout the simulation, we neglected the quantized angle error and assumed a static channel, i.e., $\mathbf{P}=\mathbf{I}$ so that algorithms developed for static channels can be applied for comparison. The receiver uses a Neyman-Person detector for sensing the paths, and the noise power at the receiver side is $\sigma_{w}^{2}=1$. SNR is the transmit power for one symbol time relative to the noise power before beamforming.

We compared the performance of the proposed POMDP strategy, the random beam selection strategy, and the CS technique based on a space bisection approach in [3]. In the random pilot beam selection strategy, one of $\left(\begin{array}{l}N_{t} \\ M_{p}\end{array}\right)$ possible pilot beam sequences is randomly selected to sense the channel at each slot. Fig. 3 shows the performance of the considered methods at two mmWave MIMO scenarios. The channel estimation performance was measured by the normalized mean square error (NMSE). In each MIMO system scenario, we set the number of the symbol times for channel estimation (i.e., $M_{p}$ ) as the smallest number of symbol times needed for the $\mathrm{CS}$ technique based on a space bisection approach in [3] (i.e., $\left.M_{p}=2 L \log _{2}\left(N_{t} / L\right)\right)$.

It is shown in Fig. 3 that the POMDP strategy significantly outperforms the random pilot beam selection strategy, and the POMDP strategy has better performance than the CS technique in [3] at the low SNR region, whereas the trend switches at the high SNR region. This is because the transmit power is scattered in the CS technique, whereas the transmit power is concentrated by pilot beamforming in the POMDP strategy. This yields better performance for the POMDP strategy at the low SNR region.

\section{CONCLUSION}

We have considered the pilot beam sequence design for sparse large mmWave MIMO channels. We have shown that the pilot beam design problem can be formulated as a POMDP problem by exploiting the sparse channel dynamic and have obtained the optimal strategy and a greedy strategy for pilot beam sequence design. The proposed pilot design method can be used to estimate the channel initially at the beginning of the transmission or to track the channel once the sparse channel locations are identified with some other method. For the initial channel identification purpose, the proposed algorithm can be modified by considering a superposed pilot beam for a pilot symbol time with adaptive resolution over slots to shorten the time for initial path location identification.

\section{REFERENCES}

[1] O. E. Ayach, R. W. Heath Jr., S. Abu-Surra, S. Rajagopal, and Z. Pi, "Low complexity precoding for large millimeter wave MIMO systems," in Proc. IEEE Int. Conf. Commun., Jun. 2012.

[2] A. Alkhateeb, O. E. Ayach, G. Leus, and R. W. Heath, "Hybrid precoding for millimeter wave cellular systems with partial channel knowledge," in Proc. ITA, (San Diego, CA), 2013.

[3] A. Alkhateeb, O. E. Ayach, G. Leus, and R. W. Heath, "Channel estimation and hybrid precoding for millimeter wave cellular systems," IEEE J. Sel. Topics Signal Process., vol. 8, pp. 831 - 846, Oct. 2014.

[4] W. U. Bajwa, J. Haupt, A. M. Sayeed, and R. Nowak, "Compressed channel sensing: a new approach to estimating sparse multipath channels," Proc. IEEE, vol. 98, pp. 1058 - 1076, Jun. 2010.

[5] G. Tauböck and F. Hlawatsch, "Compressed sensing based estimation of doubly selective channels using a sparsity-optimized basis expansion," in Proc. Eur. Signal Process. Conf., (Switzerland), Aug. 2008.

[6] J. He, T. Kim, H. Ghauch, K. Liu, and G. Wang, "Millimeter wave MIMO channel tracking systems," arXiv preprint arXiv:1412.4224, 2014.

[7] M. L. Puterman, Markov Decision Processes: Discrete Stochastic Dynamic Programming. John Wiley \& Sons, 1994.

[8] A. M. Sayeed and V. Raghavan, "Maximizing MIMO capacity in sparse multipath with reconfigurable antenna arrays," IEEE J. Sel. Topics Signal Process., vol. 1, pp. 156 - 166, Jun. 2007.

[9] A. M. Sayeed, "Deconstructing multiantenna fading channels," IEEE Trans. Signal Process., vol. 50, pp. 2563 - 2579, Oct. 2002.

[10] R. D. Smallwood and E. J. Sondik, "The optimal control of partially observable markov processes over a finite horizon," Operations Research, vol. 21, no. 5, pp. 1071-1088, 1973.

[11] E. Monahan, "State of the art - a survey of partially observable markov decision processes: Theory, models, and algorithms," Management Science, vol. 28, no. 1, pp. 1-16, 1982.

[12] S. M. Ross, Applied Probability Models with Optimization Applications. Courier Dover Publications, 1970.

[13] A. Cassandra, M. L. Littman, and N. L. Zhang, "Incremental pruning: A simple, fast, exact method for partially observable markov decision processes," in Proc. Thirteenth Ann. Conf. on Uncertainty in Artificial Intelligence, pp. 54-61, Morgan Kaufmann Publishers Inc., 1997.

[14] J. Pineua, G. Gordon, and S. Thrun, "Point-based value iteration: An anytime algorithm for POMDPs," in Proc. Int. Joint Conf. Artificial Intelligence, vol. 3, pp. 1025-1032, 2003.

[15] H. Kurniawati, D.Hsu, and W. Lee, "SARSOP: Efficient point-based POMDP planning by approximating optimally reachable belief spaces," in Proc. Robot. Sci. Syst, 2008.

[16] T. Smith and R. Simmons, "Point-based POMDP algorithms: Improved analysis and implementation," arXiv preprint arXiv:1207.1412, 2012. 